\title{
Cadaver Validation of the Use of Ultrasound for 3D Model Instantiation of Bony Anatomy in Image Guided Orthopaedic Surgery
}

\author{
C.S.K. Chan ${ }^{1}$, D.C. Barratt ${ }^{1}$, P.J. Edwards ${ }^{1,2}$, G.P. Penney ${ }^{1}$, \\ M. Slomczykowski ${ }^{3}$, T.J. Carter ${ }^{1}$, and D.J. Hawkes ${ }^{1}$ \\ 1 Imaging Sciences Division, King's College London, Guy's Hospital, London, UK \\ carolyn.chan@kcl.ac.uk \\ 2 Department of Surgical Oncology and Technology, Imperial College London, \\ London, UK \\ 3 i-Orthopaedics, DePuy a Johnson \& Johnson company, Heimstetten, Germany
}

\begin{abstract}
We present cadaver validation of a method that uses tracked ultrasound to instantiate and register 3D statistical shape models (SSMs) of 3 femurs and 2 pelves. The SSMs were generated directly from the deformation fields obtained from non-rigid registration of $\mathrm{CT}$ images to a single target CT image. Ultrasound images were obtained from three intact cadavers. These were used to instantiate the model by iteratively minimising the RMS distance between the model surface and the ultrasound-derived bone surface points. The RMS distance between the instantiated model surface and the CT-derived bone surface was less than $3.72 \mathrm{~mm}$ in the region of the femoral head and acetabulum. We conclude that SSMs of the femur and pelvis may be instantiated and registered to surgical space to within a clinically acceptable accuracy using intraoperative ultrasound. This potentially could reduce the invasiveness of orthopaedic procedures, and remove the requirement for a preoperative CT scan.
\end{abstract}

\section{Introduction}

SSMs have recently generated considerable interest for use in orthopaedic surgery, both for image segmentation and for navigation. In particular, it has been shown that a 3D surface model can be instantiated from X-ray images using an SSM [4]. We have recently proposed that instantiation of a 3D model can also be achieved using tracked ultrasound(US)[3]. The present paper describes important modifications to the method described earlier for generating the shape model, and also validation using cadaveric data where an accurate Gold Standard image-to-physical registration transformation (based on bone-implanted fiducial markers) to a high-resolution CT scan was available.

The method uses a statistical shape model (SSM) to generate an anatomically realistic model of bony anatomy given a large number of points on the bone surface extracted from US images[3]. The motivations for this work are to exploit the inherent advantages of accurate image-guided total hip replacement without 
the need for a preoperative computed tomography (CT) scan with its associated radiation dose and expense. The use of US to register the bone also results in a reduction in invasiveness of the procedure compared to conventional methods. In our previous work the SSM was built purely using bone surface points[3]. However, in the present study we have used a 3D vector field, calculated by non-rigid registration of each training $\mathrm{CT}$ image onto a template image, in order to establish correspondence between elements of the training set. This approach has the advantage that a complete reconstruction of a synthetic $3 \mathrm{D}$ volume can be achieved, including estimates of density changes and the internal structure of bony anatomy that is represented in the training data.

US provides a safe, non-invasive and relatively inexpensive method for locating bone surfaces intraoperatively. Initial phantom studies have demonstrated that US can be used to instantiate and register a femur SSM[2]. This paper describes the validation of the combined instantiation and registration of femur and pelvis SSMs using much more realistic US data collected on 3 cadavers, with corresponding CT volumes providing the ground-truth shape.

At least two other groups have developed shape models for orthopaedic surgery. Yao and Taylor 12 have demonstrated 2D/3D non-rigid registration between a statistical bone density atlas of the hip and a set of X-ray images, and instantiated a deformable hemi-pelvis model with anatomical structures visible in a CT image[11]. Fleute and Lavallee[4], and Fleute et al.[5], instantiated $3 \mathrm{D}$ anatomical surfaces of the spine from a point distribution model (PDM) with a few X-rays using a contour-based registration method. To the best of our knowledge, no other groups have attempted to instantiate a 3D model using tracked US apart from [2].

\section{Methods and Materials}

\subsection{Experimental Procedure}

Experiments for this study were carried out in the Institute of Anatomy, LudwigMaximilians University, Munich using 3 complete female cadavers preserved using the method of $\mathrm{W}$. Thiel[10]. Titanium bone screws were implanted into the femur and pelvis of each cadaver ( 4 in each femur and 5 in each hemi-pelvis) and fiducial markers, filled with CT contrast agent (Urografin 370, Scherring Health Care Ltd), were attached. A single high-resolution CT scan was then obtained for each cadaver using a spiral CT scanner (Siemans SOMATOM Plus $5)$. The extent of the CT scan included the whole pelvis and leg down to below the knee, reconstructed with voxel dimensions $0.71 \times 0.71 \times 2 \mathrm{~mm}^{3}$. Following the CT scan, each cadaver was returned to the laboratory and the contrast-filled fiducial markers were replaced with markers which had a $3 \mathrm{~mm}$ divot, the centre of which is coincident with the centroid of the fluid-filled chamber of the CT-visible markers. This enabled the CT scan to be accurately registered with physical space after using a tracked localiser to measure the 3D positions of the divot centres. All measurements were recorded relative to the $3 \mathrm{D}$ coordinate 
system of a dynamic reference object implanted into the femur or pelvis (see Fig.1).

In this study, a commercial US scanner (Philips-ATL HDI-5000) and highfrequency scan-probe (L12-5, 5-12MHz broadband linear-array transducer) were used to acquire US images. The $3 \mathrm{D}$ positions and orientations of US images were measured as the scan-probe was swept slowly across the skin surface. A high-accuracy optical tracking device (Optotrak 3020, Northern Digital Inc., Ontario, Canada) was used to track a custom-built object fixed to the scanprobe, as shown in Fig. 1. This enabled the 3D position and orientation of each $2 \mathrm{D}$ ultrasound image to be calculated with respect to the dynamic reference co-ordinate system. The use of a tracked dynamic reference object enabled the position of the cadaver to be changed during US acquisition. This allowed images to be obtained in regions that would otherwise be inaccessible with the cadaver in the supine position.

US images were collected from several approaches so that as many anatomical features as possible were scanned in order to ensure that the model instantiation was well constrained in regions such as the femoral neck and condyles. The number of US image frames acquired was between 248 and 518. The time taken to acquire them was on average 8 minutes and the total number of US points extracted was between 992 and 2107. The US images were segmented manually to extract the bone surface.
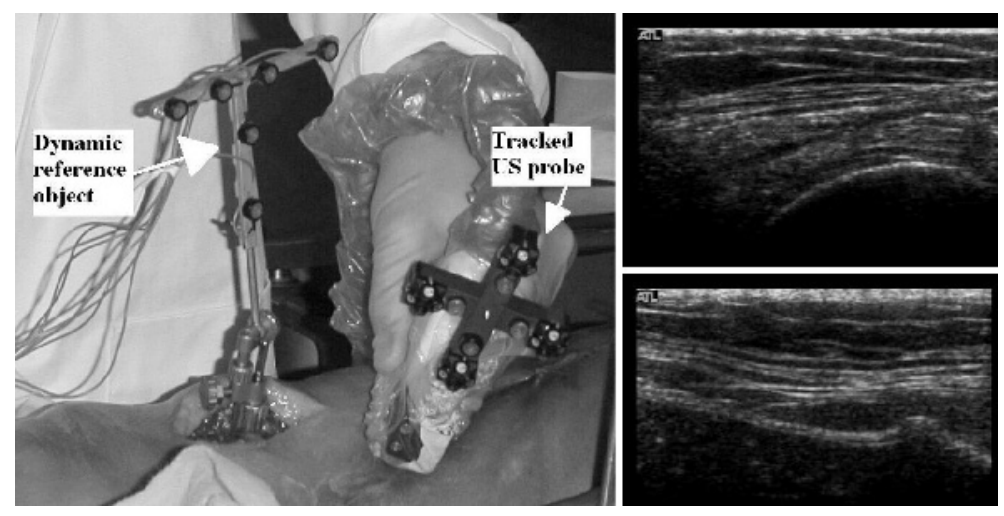

Fig. 1. Tracked US probe scanning the femur (left); sample US images in the femur (top right) and pelvis (bottom right)

\subsection{Construction of the Statistical Shape Model}

One template image was selected from our database of CT scans and aligned to the other images in the database using a non-rigid registration algorithm[8]. This 
algorithm uses a combination of global and local transformations: global motion is modelled by both rigid (rotations and translations) and affine (scaling and shearing) transformations. The shapes of the femur and pelvis are challenging for a registration algorithm based on non-rigid registration. Consequently, an initial alignment was defined manually to incorporate rigid movement and scaling (a total of nine degrees of freedom) so that the likelihood of obviously incorrect correspondences was reduced. The local transformation was then modelled by a free-form deformation (FFD) model based on B-splines[8].

The FFD has a potentially large number of degrees of freedom, and is defined by the deformed positions of a regular grid of B-spline node points. The global transformation describes the overall translation and rotation of the bone, while the local transformation is used to describe shape deformation. Normalised mutual information (NMI) was employed as a voxel-based similarity measure [9].

Using the results from the non-rigid registration described above, which registered training CT datasets to one template image, a PDM was built on the node points (also called control points) of the approximating B-splines used to define the FFD. The output of the principal component analysis (PCA) was the mean deformation of the template, with the eigenmodes and eigenvalues representing the deformation fields. The number of node points used for the femur model was about 184, whereas 395 node points were used for the pelvis model. In both cases, a node point spacing of $20 \mathrm{~mm}$ was used. This method was originally applied to magnetic resonance (MR) images of the brain with encouraging results[7].

The instantiation process generates a high-resolution, 3D grey-level image, which, in this case, has the appearance of a CT scan. In our application this allows propagation of a more densely sampled surface and incorporation of other features, such as variations in bone density, which have important implications for planning prosthesis placement in image-guided orthopaedic surgery. On the other hand, when generating a PDM purely for surface data, there is the problem of finding point correspondence, which can only be achieved by interpolating within the surface, and may give rise to errors in surface shape and texture. The method proposed here provides an estimate of all correspondences within the volume of the bone fully automatically. The femur model used in this study was built from 16 training datasets of mixed male and female femurs, whereas that of the pelvis was built from 10 training datasets of female pelves. Following PCA, the first five modes of variation were used in the model (as a compromise between computational time and population coverage), with individual variation within each mode allowed up to three standard deviations.

\subsection{Instantiation of the Statistical Shape Model}

US-derived bone surface points were matched to the corresponding SSM surface using the ICP algorithm[1], using the mean shape of each model as the starting estimate. Multiple starting positions for the rigid body registration parameters were used to initialise the instantiation process in order to simulate starting positions which could be obtained in a clinical setting. Three observers were 
invited to produce 3 starting positions, giving 9 starting positions in total for each instantiation. Given the first 5 modes of variation, the root-mean-square(RMS) point-to-surface distance was then minimised using a multiple layer optimisation strategy. In this scheme, the weight corresponding to each mode of variation was varied and the US points registered to the resulting shape model using the ICP algorithm. A four layer strategy was used, where two modes were considered at layer 1 (modes 1 and 2) and this was increased to five modes by layer 4 (modes 1 to 5). On each iteration, a Golden Section search [6] was used to optimise the shape within one mode alone, with the weight corresponding to all other modes held constant. Visual inspection revealed that one of the pelvic datasets had a number of US points which were obvious outliers. These were removed by aligning the US points with the mean shape and then excluding $5 \%$ of the points with the largest RMS point-to-surface distance were excluded from the instantiation.

\section{$3 \quad$ Results}

Fig. 2 shows the first mode for the left femur and pelvis models when applying plus or minus three standard deviations to the mean shape. The first mode of the left femur model can be seen to correspond to a change in the femur size and length. Other significant modes of variations include a twisting of the femoral head angle and change of trochanter and condyle sizes.
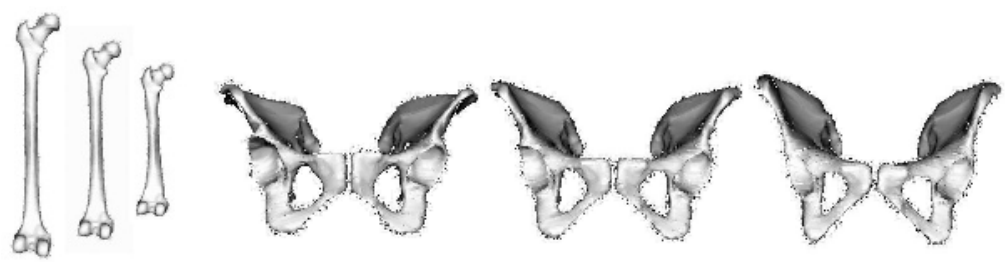

Fig. 2. The left femur PDM (left) and the pelvis PDM (right) showing the first mode of variation with $+/$ - three standard deviations.

The results of the femur and pelvis instantiations from cadaver 1 are illustrated in Fig. 3 and 4. In each figure, the bone surface segmented from the CT scan was used as the Gold Standard shape model. Results from other cadavers were similar.

Numerical results are given in Table 1 calculated in three different ways. Firstly, the RMS distance was calculated between the instantiated SSM and the US-derived bone surface points registered using the ICP algorithm. Secondly, the RMS distance was calculated between the instantiated SSM and the CTderived surface, using the Gold Standard (GS) transformation, calculated from point-based registration of the bone-implanted fiducial markers. This measure 


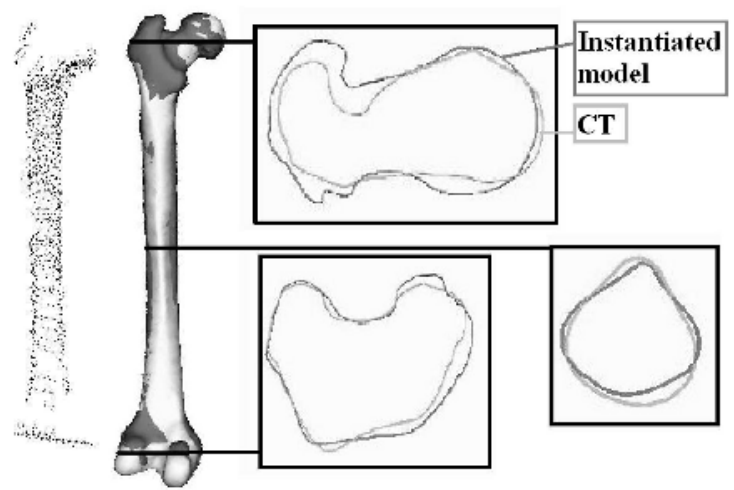

Fig. 3. (Left to right) US-derived femur surface points (left) and the instantiated SSM overlaid on the CT-derived surface, shown in dark grey. Cross-sectional contours from the instantiated femur model (black) with corresponding contours from the CT (grey). From top to bottom: the condyles; mid-shaft; and the femoral head.

indicates the combined accuracy of the geometry of the instantiated model and its registration in physical space. Thirdly, the ICP algorithm was used to register the instantiated model and CT surface to provide a measure of the geometric accuracy of the instantiated shape.

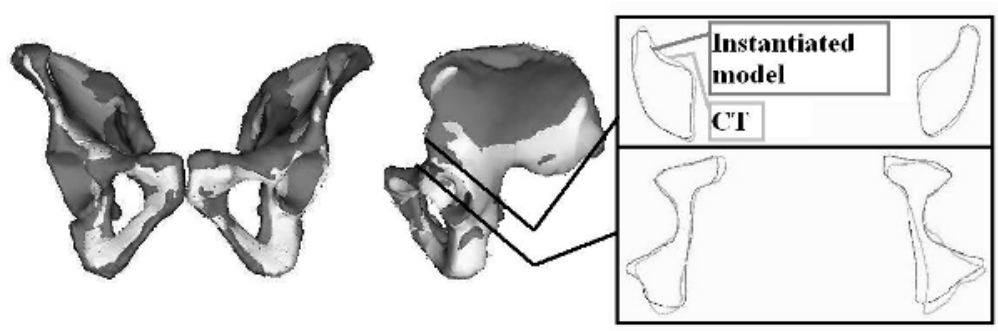

Fig. 4. (Left to right) The instantiated SSM (frontal and lateral views) overlaid on the CT-derived surface, shown in dark grey. Cross-sectional contours from the instantiated pelvis model (black) with corresponding contours from the CT (grey). From top to bottom: near top of acetabulum and middle of acetabulum.

The RMS distance after registering the instantiated model and US points was between 1.52 and $1.96 \mathrm{~mm}$ for the femur, and between 2.47 and $4.23 \mathrm{~mm}$ for the pelvis. Since the application area of this study is hip replacement surgery, an RMS distance error was also deter-mined in the region of most clinical relevance: In the femur the RMS distance errors were recomputed in the region of the 
femoral head and neck, and greater and lesser trochanters, yielding an RMS error in the range 1.83 to $3.69 \mathrm{~mm}$ for GS and 1.83 to $3.64 \mathrm{~mm}$ for ICP (See Table 1). In the pelvis, the region around the acetabulum was chosen as having most clinical relevance, with a resulting RMS error in the ranges 3.62 to $3.72 \mathrm{~mm}$ for GS and 2.61 to $3.71 \mathrm{~mm}$ for ICP. In each case, all of the 9 starting positions converged to the same RMS distance within 3 significant figures.

Table 1. Average RMS distance for instantiated femur and pelvic surfaces: * Distance between US-derived bone surface points and the instantiated SSM surface; ${ }^{* *}$ distance between the vertices of the surface mesh, which describes the instantiated SSM surface, and the CT-derived bone surface calculated after (i) transforming the CT surface to physical space using the Gold Standard, fiducial-based registration (GS) and (ii) aligning the datasets using the ICP algorithm (ICP); *** as for ** above, but with the measure restricted to regions of clinical interest.

\begin{tabular}{l|c|c|c|c|c}
\hline \hline Cadaver \& bone & $\begin{array}{c}\text { US-Model } \\
(\mathrm{mm}) *\end{array}$ & \multicolumn{2}{|c|}{$\begin{array}{c}\text { CT-Model } \\
(\text { global })(\mathrm{mm}) * *\end{array}$} & \multicolumn{2}{c}{$\begin{array}{c}\text { CT-Model } \\
(\text { regional })(\mathrm{mm})^{* * *}\end{array}$} \\
\hline & ICP & GS & ICP & GS & ICP \\
\hline 1 (femur) & 1.96 & 3.26 & 2.17 & 2.90 & 2.46 \\
\hline 2 (femur) & 1.54 & 2.65 & 1.88 & 1.83 & 1.83 \\
\hline 3 (femur) & 1.52 & 3.05 & 2.71 & 3.69 & 3.64 \\
\hline 1 (pelvis) & 4.23 & 4.95 & 2.93 & 3.62 & 2.61 \\
\hline 3 (pelvis) & 2.47 & 5.00 & 4.88 & 3.72 & 3.71 \\
\hline \hline
\end{tabular}

\section{Discussion and Conclusion}

This work presents the use of US images to instantiate and register a statistical shape model constructed using FFD fields produced using non-rigid registration. In conclusion, preliminary analysis of the data obtained on cadavers suggests that SSMs of the femur and pelvis can be simultaneously instantiated and registered to surgical space with a clinically useful accuracy using intraoperative US data ( $<3.72 \mathrm{~mm}$ RMS for both the femoral head and acetabulum).

One advantage of models produced using FFD fields as opposed to surface points is that they provide much higher resolution. Further work is required to establish this relationship which may then be input as a prior into an automated algorithm. Our plan is to extend this algorithm to one that automatically aligns the US-derived surfaces to the CT surfaces of the model, but to achieve this we need a more accurate model of the precise relationship between the US and CT bone surfaces. Future work includes further automation of the process of US segmentation, and further development of model building and instantiation processes, perhaps including one or two X-rays as additional input data. An algorithm has been suggested by Fleute et al. [4] to reconstruct bones by registering a PDM to X-ray views. One strategy that could be explored is to instantiate 
the model from one or two X-rays and register using US. Finally, data from the remaining 3 femurs and 1 pelvis is currently being processed and will be reported in due course.

Acknowledgements. This project is funded by the Engineering and Physical Sciences Research Council (EPSRC), United Kingdom (GR/R03525/01 in collaboration with DePuy International and Brainlab AG). The authors wish to thank DePuy International for providing the femur images that were used to build the shape model, Professor Dr. med. R. Putz, Anatomische Anstalt, Ludwig-Maximilian-Universität, München (LMU) for the cadavers and facilities for the cadaver experiments and Philips Medical Systems for advice on US. We also thank the staff of the Radiology Department at LMU for their assistance with CT scanning.

\section{References}

1. Besl, P.J., McKay, N.D.: A method for registration of 3D shapes. IEEE Transactions on Pattern Analysis and Medicine Intelligence 14 (1992) 239-256

2. Chan, C.S.K. et al.: Ultrasound-based reconstruction and registration of 3D bone anatomy using statistical shape models. Proceedings of CAOS International, Chicago (2004)

3. Chan, C.S.K., Edwards, P.J., Hawkes, D.J.: Integration of ultrasound based registration with statistical shape models for computer assisted orthopaedic surgery. Proceedings of SPIE Medical Imaging 2003: Image Processing 5032 (2003) 414-424

4. Fleute, M., Lavallee, S.: Nonrigid 3D/2D registration of images using statistical models. Proceedings of MICCAI (1999) 138-147

5. Fleute, M., Lavallee S., Desba, L.: Integrated approach for matching statistical shape models with intra-operative 2D and 3D data. Proceedings of MICCAI (2002) 365-372

6. Press, W.H., Teukolsky, S.A., Vetterling, W.T., Flannery, B.P.: Minimization or Maximization of Functions. In: Numerical Recipes in C, 2nd edn. Cambridge University Press, Cambridge (1992) 394-455

7. Rueckert, D., Frangi, A.F., Schnabel, J.A.: Automatic construction of 3D statistical deformation models using non-rigid registration. Proceedings of MICCAI (2001) $77-84$

8. Rueckert, D. et al.: Nonrigid registration using free-form deformations: application to breast MR images. IEEE Transactions on Medical Imaging. 18 (1999) 712-721

9. Studholme, C., Hill, D.L.G., Hawkes, D.J.: An overlap invariant entropy measure of 3D medical image alignment. Pattern Recognition 32 (1999) 71-86

10. Thiel, W.: Ergänzung für die konservierung ganzer leichen nach W. Thiel. Annals of Anatomy 184 (2002) 267-270

11. Yao, J., Taylor, R.: A multiple-layer flexible mesh template matching method for nonrigid registration between a pelvis model and CT images. Proceedings of SPIE Medical Imaging: Image Processing 5032 (2003) 1117-1124

12. Yao, J., Taylor, R.: Deformable registration between a statistical bone density atlas and X-ray Images. Proceedings of CAOS International, Santa Fe (2002) 168-169 